Research Paper

\title{
Investigating a Correlation between Chemoradio- therapy Schedule Parameters and Overall Survival in a real-life LD SCLC Patient Cohort
}

\author{
Farkhad Manapov ${ }^{1^{\star} \bowtie}$, Chukwuka Eze ${ }^{1^{*}}$, Maximilian Niyazi ${ }^{1}$, Olarn Roengvoraphoj ${ }^{1}$, Minglun Li ${ }^{1}$, \\ Nina-Sophie Hegemann1, Guido Hildebrandt², Rainer Fietkau³, Claus Belka1,4 \\ 1. Department of Radiation Oncology, LMU Munich, Marchioninistrasse 15, 81377, Munich, Germany. \\ 2. Department of Radiation Oncology, University of Rostock, Südring 75, 18059, Germany. \\ 3. Department of Radiation Oncology, Friedrich-Alexander University Erlangen-Nuernberg, Universitätsstrasse 27, 91054, Erlangen, Germany. \\ 4. Comprehensive Pneumology Center, The German Center for Lung Research, Munich, Germany. \\ * Farkhad Manapov and Chukwuka Eze contributed equally to this work.
}

$\triangle$ Corresponding author: Dr. med. Farkhad Manapov, Klinik und Poliklinik für Strahlentherapie und Radioonkologie, Klinikum der Universität München Marchioninistrasse 15, 81377 München, Germany. E-mail: Farkhad.Manapov@med.uni-muenchen.de; dermatom@mail.ru Tel.: +49/89-440073770 Fax.: $+49 / 89-440076770$.

(C) Ivyspring International Publisher. Reproduction is permitted for personal, noncommercial use, provided that the article is in whole, unmodified, and properly cited. See http://ivyspring.com/terms for terms and conditions.

Received: 2016.07.06; Accepted: 2016.09.18; Published: 2016.10.17

\begin{abstract}
Background: Chemoradiotherapy (CRT) is a treatment standard in limited disease (LD) small cell lung cancer (SCLC). Currently, the timing of thoracic radiation therapy (TRT) remains the subject of randomised trials and meta-analyses. To investigate a correlation between CRT schedule parameters and overall survival (OS) in a real-life patient cohort, a temporal analysis was performed.

Methods: 182 LD SCLC patients successfully treated with definitive CRT were retrospectively reviewed. TRT was applied concurrently or sequentially. Impact of the treatment mode and interval of simultaneous treatment (IST) (an interval in days when chemotherapy and TRT were applied simultaneously, including time between chemotherapy cycles and weekends) on OS was analysed.

Results: 71 (39\%) patients were treated with concurrent and 111 (61\%) with sequential CRT. Median overall survival (MS) for the entire cohort was 534 days $(95 \% \mathrm{Cl} 461-607)$ without any significant difference between the concurrent and sequential groups (589: $95 \% \mathrm{Cl} 358$ - 820 vs. $533: 95 \% \mathrm{Cl} 446$ 620 days, $\mathrm{p}=0.746$, log-rank test). IST was 0 days in 111 (61\%) patients treated sequentially whereas in the concurrent group, 20 (11\%) and 51 (28\%) patients showed an IST < 35 and > 35 days, respectively. Patients with IST $>0$ and $<35$ days demonstrated a trend to improved overall survival (MS: IST 0 vs. > 35 vs. $<35$ was 533 vs. 448 vs. 1169 days, $p=0.109$, log-rank test). When patients treated with sequential CRT (IST 0) were excluded from the analysis, statistical difference in overall survival according to the IST subgroups (IST $>35$ vs. $<35$ ) became significant $(p=0.021$, log-rank test). On multivariate analysis of patients treated with concurrent CRT, IST $>0$ and $<35$ days remained a variable that significantly correlated with better overall survival ( $p=0.039$, HR 0.38).

Conclusion: In this real-life LD SCLC patient cohort, improved overall survival was achieved in patients treated with CRT schedule according to the IST $>0$ and $<35$-day concept. By exceeding the 35-day interval, we have seen deterioration in survival.
\end{abstract}

Key words: small-cell lung cancer, limited disease, chemoradiotherapy, thoracic radiation therapy.

\section{Introduction}

Lung cancer is the leading cause of highest incidence in both genders. SCLC is a highly cancer-related death worldwide with the second aggressive neoplasia and accounts for $13 \%$ to $15 \%$ of 
total lung cancer diagnoses [1]. SCLC is characterised by rapid doubling time, early systemic dissemination and high sensitivity to chemo- and radiotherapy [2-4]. At initial diagnosis, only $30 \%$ patients present with LD. Actual median survival and a 2-year survival rate in patients with LD varies from 15 to 20 months and $20 \%$ to $40 \%$, respectively [5].

Due to rapid loco-regional failures after chemotherapy alone, the adjunction of TRT was investigated and showed improved local control and better long-term outcome [6-8]. Hence, multimodality treatment consisting of platinum-based chemotherapy and TRT has become the standard of care $[5,9,10]$. Multiple clinical trials and meta-analyses addressing the issue of timing of TRT have been published, with the weight of evidence suggesting a small benefit for early TRT (i.e. TRT administered during the first or second cycle of chemotherapy) [11-21]. The meta-analysis by De Ruysscher et al. revealed that a short time between the first day of any treatment and the last day of TRT is associated with improved OS [21]. However, the latest published randomised phase III study investigating the timing of TRT during the course of chemotherapy in LD SCLC found no differences in the remission rates and overall survival between early (starting with first) and late (starting with the third cycle of chemotherapy) irradiation groups [22]. Our previous study in LD SCLC demonstrated that short and dose-dense CRT correlated with improved overall survival in patients with poor initial performance status (PS) [23].

The aim of the present analysis was to evaluate an impact of the chemoradiotherapy schedule parameters on overall survival in a real-life heterogeneous patient cohort and define a role of IST as a treatment related prognostic factor.

\section{Patients and Methods}

182 patients from two institutions in Germany with initial PS score WHO 0-3 were diagnosed with LD (UICC Stage I-IIIA/B) SCLC and successfully treated with definitive CRT in the time from 1998 to 2012. Diagnosis was histologically confirmed in all patients. LD was defined according to Murray et al. as disease confined to one hemithorax with or without contralateral mediastinal and ipsilateral supraclavicular lymph node involvement [24]. Evidence of pleural effusion and involvement of the contralateral supraclavicular and/or hilar lymph nodes was considered to be an exclusion criterion [25]. In all patients initial staging included bronchoscopy with biopsy, computed tomography (CT) scans of the chest and abdomen, bone scintigraphy and first contrast-enhanced cranial magnetic resonance imaging (MRI). All patients provided written informed consent prior to commencement of primary treatment. CRT was applied concurrently in seventy-one $(39 \%)$ patients and consisted of TRT starting with the first or second cycle of chemotherapy followed by consolidation cycles. One hundred and eleven $(61 \%)$ patients were treated sequentially with four to six chemotherapy cycles followed by TRT. Chemotherapy was given in a 28-day cycle in patients treated with concurrent and in a 21-day cycle in patients treated with sequential CRT according to Takada et al. [14]. TRT was delivered by linac with megavoltage capability (8-15 MV) using coplanar multiple field technique. Three-dimensional CT-simulated treatment planning was performed in all patients. Planning target volume was defined as a primary tumour bulk including involved lymph nodes visualised on the pre-therapeutic CT with $1.0 \mathrm{~cm}$ margin. $96 \%$ of the patient cohort was treated 5 days a week with conventional fractionation to a total dose of at least 50.0 Gy (range: 50.0 - 66.0 Gy). $4 \%$ of patients were treated with hyperfractionated accelerated TRT according to Turrisi et al. [18]. Second contrast-enhanced cranial MRI was routinely performed before commencing PCI to exclude BM according to Manapov et al. [26]. PCI was applied with a daily fraction of 1.8/2.0 Gy to a total dose of 30.0 to 36.0 Gy. Patients were followed every 3 months for the first two years and every 6 months thereafter. Response evaluation was performed by a radiologist based on CT of the thorax and abdomen. Other imaging techniques including FDG-PET/CT were applied if clinically indicated.

Due to the fact that the majority of patients died, there is no median follow-up; hence, follow-up was as complete as possible. Survival rates were measured from initial diagnosis. Kaplan-Meier analyses (pair-wise comparisons) were used to compare survival curves for subgroups according to the definition of IST (IST 0 vs. > 35 vs. < 35). IST was also analysed for its association with OS by multivariate Cox regression after adjustment for other prognostic factors.

\section{Results}

\section{Patient- and Treatment Characteristics}

In total, the medical charts of 182 patients were reviewed (See Table 1 for Patients and treatment characteristics). Of 182 patients treated, $110(60 \%)$ were men and $72(40 \%)$ were women. Median age at diagnosis was 63 years (range: 35 - 83). 34 (19\%) patients were older than 70 years. Median PS according to $\mathrm{WHO}$ for the entire cohort was 1 (range: 0 to 3). 71 (39\%) patients were treated with CRT concurrently vs. $111(61 \%)$ sequentially. There were 
no significant differences in regard to age, sex, PS and TNM-stage between both CRT groups. Platinum-based chemotherapy was applied in 162 $(89 \%)$ patients. $36(20 \%)$ patients were treated with less than four cycles of chemotherapy. PCI was applied in 71 (39\%) patients, who achieved partial or complete remission. Median duration of TRT was 43 days and was equal in both groups. Median duration of chemotherapy was 128 and 93 days in the concurrent and sequential groups, respectively.

\section{Definition of The Interval of Simultaneous Treatment}

IST was defined as an interval in days when chemotherapy and TRT were applied simultaneously, including time between chemotherapy cycles and weekends (Figure 1). Possible IST variants referring to the applied CRT schedules in our patient cohort are illustrated in Figure 2. Different IST values (30, 35, 42 and 49 days) have been tested. IST 35 was analysed after consideration of the TRT duration including a subset of patients treated according to Turrisi et al. and patient distribution in the different IST subgroups. Also a relevant point was that IST 35 included two completed chemotherapy cycles applied concurrently to TRT. The start of TRT during the course of chemotherapy was not the subject of this investigation. All patients treated with sequential CRT were referred to the IST 0 subgroup.

\section{Interval of Simultaneous Treatment and Overall Survival}

Median overall survival (MS) for the entire treated cohort was 534 days $(95 \%$ CI 461 - 607) without a significant difference between the concurrent (589: $95 \% \mathrm{CI} 358$ - 820) and sequential groups (533: 95\%CI 446 - 620) days) ( $p=0.746$, log-rank test). IST was 0 days in $111(61 \%)$ patients treated sequentially whereas in the concurrent group $20(11 \%)$ and 51 $(28 \%)$ patients showed an IST $<35$ and $>35$ days, respectively. Evaluation of the entire cohort demonstrated that patients treated with IST $>0$ and $<$ 35 days showed a trend to improved overall survival (IST 0 vs. > 35 vs. < 35 was 533: 95\%CI 446 - 620 vs. 448: 95\%CI 361 - 535 vs. 1169: 95\%CI 800 - 1538 days, $\mathrm{p}=0.109$, log-rank test) (See Figure 3 and Table 2). When patients treated sequentially (IST 0) were excluded from the analysis, statistical difference in overall survival achieved significance $(p=0.021$, log-rank test). On multivariate analysis of patients treated with concurrent CRT, IST $>0$ and $<35$ days remained a significant variable correlating with better overall survival ( $p=0.039$, HR 0.38$)$.

Table 1: Patients and treatment characteristics

\begin{tabular}{ll}
\hline $\begin{array}{l}\text { Gender } \\
\text { Male }\end{array}$ & $\mathrm{n}=110(60 \%)$ \\
$\begin{array}{l}\text { Female } \\
\text { Age }\end{array}$ & $\mathrm{n}=72(40 \%)$ \\
$\begin{array}{l}\text { Median } 63 \text { years (range: } 35-83) \\
>70\end{array}$ & $\mathrm{n}=34(19 \%)$ \\
$\begin{array}{l}\text { Chemoradiotherapy } \\
\text { concurrent } \\
\text { sequential }\end{array}$ & $71(39 \%)$ \\
PCI & $111(61 \%)$ \\
yes & \\
no & \\
Platinum-based chemotherapy & $\mathrm{n}=71(39 \%)$ \\
yes & $\mathrm{n}=111(61 \%)$ \\
no & $\mathrm{n}=162(89 \%)$ \\
IST subgroups & $\mathrm{n}=20(11 \%)$ \\
IST 0 days & \\
IST $>0$ and $<35$ days & $111(61 \%)$ \\
IST $>35$ days & $20(11 \%)$
\end{tabular}

Table 2: Median overall survival rates according to IST

\begin{tabular}{lc}
\hline IST definition in days & Overall survival in days $(95 \% \mathrm{CI})$ \\
\hline IST 0 & 533 \\
& $95 \% \mathrm{CI} 446-620$ \\
IST $>0$ and $<35$ & 1169 \\
& $95 \% \mathrm{CI} 800-1538$ \\
IST $>35$ & 448 \\
& $95 \%$ CI $361-535$ \\
\hline
\end{tabular}

IST 0

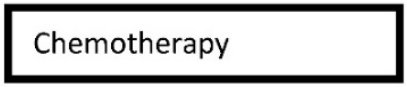

\section{Radiotherapy}

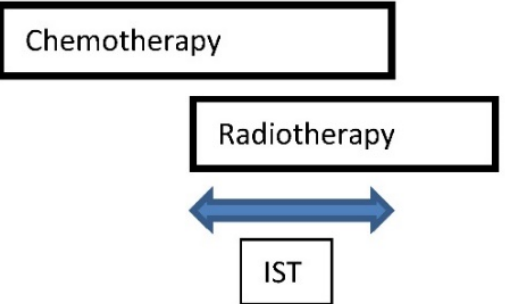

Chemotherapy

Radiotherapy

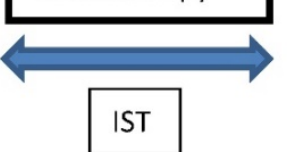

Figure 1: Definition and Variations of IST 


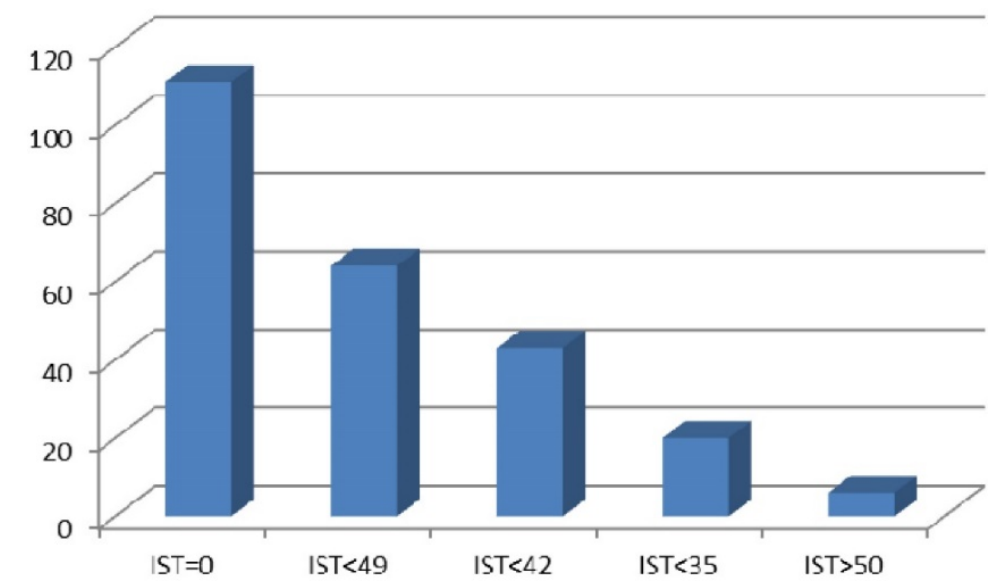

Figure 2: IST variants in the patient cohort

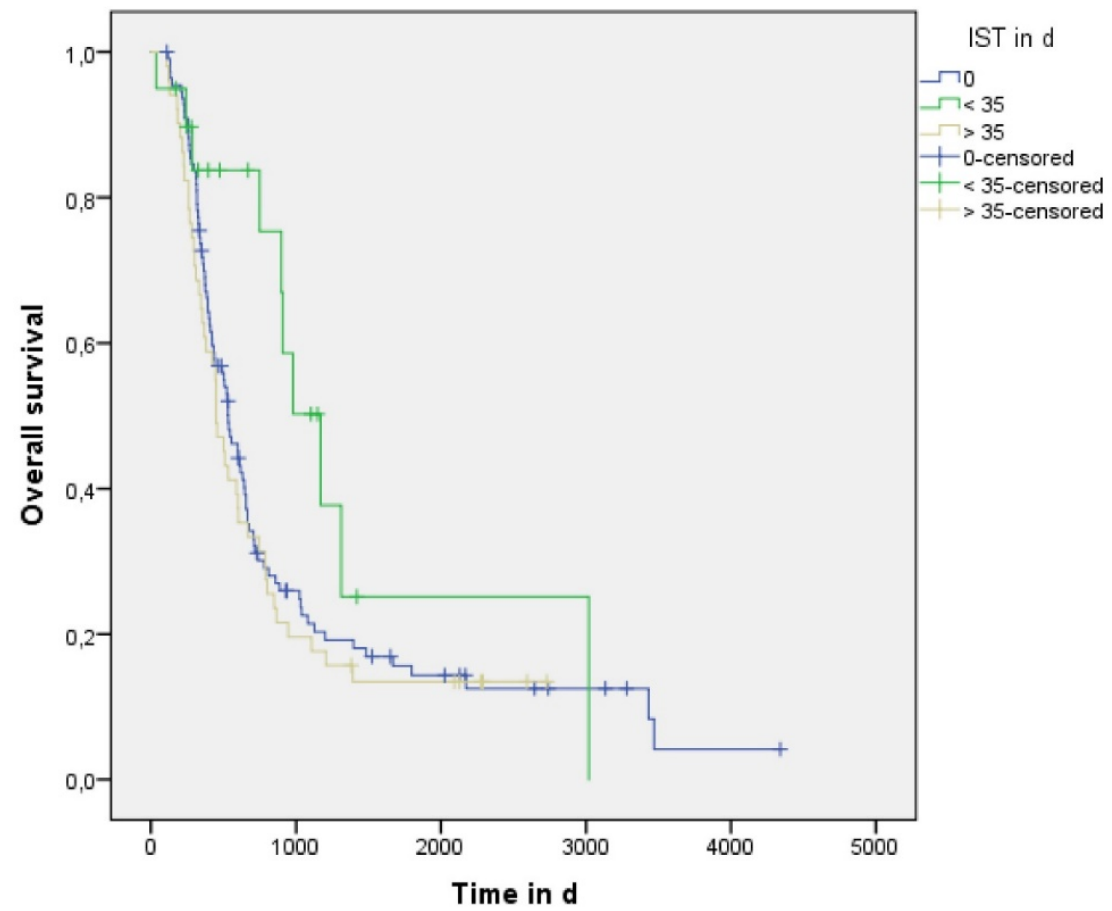

Figure 3: Overall survival in patient subgroups defined by IST

\section{Discussion}

The main finding of this temporal CRT schedule analysis of a real-life heterogeneous LD SCLC cohort is that improved overall survival was attained when both chemotherapy and TRT were applied simultaneously in the multimodality treatment course in a time interval not exceeding 35 days (IST $>0$ and $<$ 35). Importantly, application of CRT schedule according to the IST $>0$ and $<35$ concept has led to more than a doubling of the MS compared to the rest of the treated cohort. Another relevant point was that the start of TRT (early vs. late) during the course of multimodality treatment was not a limiting factor but rather a subject of this investigation.
CRT schedule is considered an important treatment-related factor in LD SCLC since several meta-analyses reported significantly improved survival rates when early platinum-based concurrent CRT is applied and overall treatment time of TRT is less than 30 days. Furthermore, the meta-analysis by De Ruysscher et al. revealed that short time between the first day of chemotherapy and the last day of TRT (SER) is an important predictor of survival and defined a short SER ( $<30$ days) as a pre-requisite for favourable long-term outcome. The novel parameter SER was established to take into account accelerated proliferation of the tumour clonogens during both radio- and chemotherapy. The authors concluded that SER might facilitate the design of future 
combined-modality treatment trials [21]. The role of short SER (<30 days), however, was not confirmed in the last published randomised phase III trial investigating the timing of TRT. The study could not find any differences in the remission rate, progression-free and overall survival between early (starting with the first) and late (starting with the third chemotherapy cycle) irradiation arms [22]. These results have excited a discussion about the exact definition of short SER. A small korean study defined short SER as less than 60 days and reported a correlation between short SER and improved survival [27]. Another relevant aspect is the utility of hyperfractionated accelerated CRT in daily clinical practice. A previous analysis by Komaki et al. showed that $21 \%$ of LD SCLC patients from 39 institutions in the United States treated from 2006-2007 received twice-daily CRT according to the Turrisi et al. protocol [28]. In this present cohort, the rate was much lower (4\%). Furthermore, our previous analysis demonstrated that duration of CRT, itself, correlated with overall survival in limited LD SCLC patients with poor initial PS [23]. Considering all of these points, we decided to analyse a value of IST and not SER in our heterogeneous cohort.

Two major limitations of the present investigation are its retrospective nature and an absence of toxicity analysis. Hence, comparison of treatment-related morbidity in the various IST subgroups was not possible. According to previous data, a higher rate of acute toxicity in CRT protocols with short SER is strongly supposed [16,20,21]. Additional analysis of the value of IST in the multimodality treatment of LD SCLC will come from two ongoing randomised phase III trials that compare different TRT protocols $[29,30]$. The final publications of both trials are eagerly awaited.

\section{Conclusion}

IST as a new treatment-related parameter can be referred simultaneously to the dose-intensity and patient compliance during the course of chemoradiotherapy. Our temporal analysis of CRT schedule in a real-life LD SCLC patient cohort found that IST $>0$ and $<35$ days significantly correlates with improved overall survival. By exceeding this interval, we have seen deterioration in patient long-term outcome.

\section{Acknowledgement}

Sabine Kloecking of the cancer registry of the University Hospital Rostock for her assistance in the data collection.

\section{Authors' Contributions}

All persons listed as authors have read the manuscript and given their approval for the submission. FM collected data, participated in the study design, performed the statistical analysis, wrote the manuscript. CE collected data, participated in the study design, wrote the manuscript. MN performed the statistical analysis, edited the manuscript. OR collected data, wrote the manuscript. ML edited the manuscript. NSH edited the manuscript. GH collected data, participated in the study design. RF collected data, participated in the study design. CB participated in the study design, edited the manuscript.

\section{Competing Interests} interests.

The authors declare they have no competing

\section{References}

1. Govindan R, Page N, Morgensztern D et al. Changing epidemiology of small-cell lung cancer in the United States over the last 30 years: analysis of the Surveillance, Epidemiologic, and End Results database. J ClinOncol. 2006;24:4539-4544. doi: 10.1200/JCO.2005.04.4859.

2. Brade AM, Tannock IF. Scheduling of radiation and chemotherapy for limited-stage small-cell lung cancer: Repopulation as a cause of treatment failure? J ClinOncol. 2006;24:1020-1022. doi: 10.1200/JCO.2005.04.9676.

3. Jackman DM, Johnson B. Small-cell lung cancer. Lancet. 2005;366:1385-1396. doi: 10.1016/S0140-6736(05)67569-1.

4. Stupp R, Monnerat C, Turrisi AT. Small cell lung cancer: state of the art and future perspectives. Lung Cancer. 2004;45:105-117. doi: 10.1016/j.lungcan.2003.12.006.

5. van Meerbeeck JP, Fennell DA, De Ruysscher D. Small cell lung cancer. Lancet 2011;378:1741-55.

6. Payne DG, Arriagada R, Dombernowsky P et al. The role of thoracic radiation therapy in small cell carcinoma of the lung: a consensus report. Lung Cancer 1989;5:135-8.

7. Warde P, Payne D. Does thoracic irradiation improve survival and local control in limited stage small cell carcinoma of the lung - a meta-analysis. Int J Radiat Oncol Biol Phys 1991;19(Suppl 1):146. abstract.

8. Pignon JP, Arriagada R, Ihde DC et al. A meta-analysis of thoracic radiotherapy for small-cell lung cancer. N Engl J Med. 1992 Dec 3;327(23):1618-24. PubMed PMID:1331787.

9. Stahel R, Thatcher N, Fruh M et al. 1st ESMO Consensus Conference in lung cancer; Lugano 2010: small-cell lung cancer. Ann Oncol 2011;22:1973-80.

10. [Internet] NCCN Clinical Practice Guidelines in Oncology: Small Cell Lung Cancer, version 1.2016. https://www.nccn.org/professionals/ physician_gls/pdf/sclc.pdf.

11. Murray N, Coy P, Pater JL et al. Importance of timing for thoracic irradiation in the combined modality treatment of limited-stage small-cell lung cancer. The National Cancer Institute of Canada Clinical Trials Group. J Clin Oncol, 1993; 11 (2): 336-44.

12. Turrisi AT 3rd, Glover DJ: Thoracic radiotherapy variables: influence on local control in small cell lung cancer limited disease. Int J Radiat Oncol Biol Phys, 1990; 19 (6): 1473-9.

13. McCracken JD, Janaki LM, Crowley JJ et al. Concurrent chemotherapy/radiotherapy for limited small-cell lung carcinoma: a Southwest Oncology Group Study. J Clin Oncol, 1990; 8 (5): 892-8.

14. Takada M, Fukuoka M, Kawahara M et al. Phase III study of concurrent versus sequential thoracic radiotherapy in combination with cisplatin and etoposide for limited-stage small-cell lung cancer: results of the Japan Clinical Oncology Group Study 9104. J Clin Oncol, 2002; 20 (14): 3054-60.

15. Spiro SG, James LE, Rudd RM et al. Early compared with late radiotherapy in combined modality treatment for limited disease small-cell lung cancer: a London Lung Cancer Group multicenter randomized clinical trial and meta-analysis. J Clin Oncol, 2006; 24 (24): 3823-30.

16. De Ruysscher D, Pijls-Johannesma M, Vansteenkiste J et al. Systematic review and meta-analysis of randomised, controlled trials of the timing of chest radiotherapy in patients with limited stage, small-cell lung cancer. Ann Oncol, 2006; 17 (4): 543-52.

17. Fried DB, Morris DE, Poole C et al. Systematic review evaluating the timing of thoracic radiation therapy in combined modality therapy for limited-stage small-cell lung cancer. J Clin Oncol, 2004; 22 (23): 4837-45. 
18. Turrisi AT 3rd, Kim K, Blum R et al. Twice-daily compared with once-daily thoracic radiotherapy in limited small-cell lung cancer treated concurrently with cisplatin and etoposide. N Engl J Med, 1999; 340 (4): 265-71.

19. Huncharek M, McGarry R: A meta-analysis of the timing of chest irradiation in the combined modality treatment of limited-stage small cell lung cancer. Oncologist, 2004; 9 (6): 665-72.

20. Pijls-Johannesma MC, De Ruysscher D, Lambin P et al. Early versus late chest radiotherapy for limited stage small cell lung cancer. Cochrane Database Syst Rev, 2005;1: CD004700.

21. De Ruysscher D, Pijls-Johannesma M, Bentzen SM et al. Time between the first day of chemotherapy and the last day of chest radiation is the most important predictor of survival in limited-disease small-cell lung cancer. J ClinOncol. 2006 Mar 1;24(7):1057-63. PubMed PMID: 16505424.

22. Sun JM, Ahn YC, Choi EK et al. Phase III trial of concurrent thoracic radiotherapy with either first- or third-cycle chemotherapy for limited-disease small-cell lung cancer. Ann Oncol. 2013 Aug;24(8):2088-92. doi: 10.1093/annonc/mdt140. Epub 2013 Apr 16. Erratum in: Ann Oncol. 2014 Aug;25(8):1672. PubMed PMID: 23592701

23. Manapov F, Klöcking S, Niyazi $M$ et al. Chemoradiotherapy duration correlates with overall survival in limited disease SCLC patients with poor initial performance status who successfully completed multimodality treatment. Strahlenther Onkol. 2012 Jan;188(1):29-34. doi:10.1007/s00066-011-0016-9. Epub 2011 Dec 23. PubMed PMID: 22189436.

24. Murray N, Coy P, Pater JL et al. Importance of timing for thoracic irradiation in the combined modality treatment of limited stage small-cell lung cancer. J ClinOncol. 1993;11:336-44.

25. Sherman CA, Rocha Lima CM, Turrisi AT. Limited small-cell lung cancer: a potentially curable disease. Oncology (Williston Park). 2000;14(10):1395-403.

26. Manapov F, Klautke G, Fietkau R. Prevalence of brain metastases immediately before prophylactic cranial irradiation in limited disease small cell lung cancer patients with complete remission to chemoradiotherapy: a single institution experience. J ThoracOncol 2008;3(6): 652-655.

27. Lee KJ, Lee EJ, Hur GY et al. The start of chemotherapy until the end of radiotherapy in patients with limited-stage small cell lung cancer. Korean J Intern Med. 2013 Jul;28(4):449-55.

28. Komaki R, Khalid N, Langer CJ et al. Penetration of recommended procedures for lung cancer staging and management in the United States over 10 years: a quality research in radiation oncology survey. Int J Radiat Oncol Biol Phys. 2013 Mar 15;85(4):1082-9.

29. [Internet] https://clinicaltrials.gov/show/NCT00433563.

30. [Internet] https://clinicaltrials.gov/show/NCT00632853. 